\title{
ESPE Working Groups
}

tic peptide hormone (drug), as a transcriptional target of SHOX. The ability of SHOX to transactivate the NPPB endogenous promoter was demonstrated in luciferase reporter assays using serial deletions of the NPPB promoter region. Binding of SHOX to the NPPB promoter was also demonstrated in vivo by chromatin fixation and immunoprecipitation. We also demonstrate the lack of promoter activation in two SHOX mutants from patients with Léri-Weill syndrome. In addition, immunohistochemical analysis of human growth plate sections showed for the first time a co-expression of BNP and SHOX in late proliferative and hypertrophic chondrocytes. Together these data strongly suggest that BNP represents a direct target of SHOX. One may speculate that BNP as a downstream effector of SHOX may also open up new potential avenues for the treatment of short stature. BNP in the systemic circulation is likely to reach growth plate chondrocytes. It may either directly influence NPR-B signaling or indirectly increase local CNP levels by saturating the competing receptor NPR-C. As demonstrated in the mouse, increased levels of BNP in the serum increase skeletal growth, maybe analogous to the use of recombinant growth hormone and insulin-like growth factor.

WG1-45 Joint Session of the ESPE Bone Club and the ESPE Growth Plate Working Group

C-type natriuretic peptide and longitudinal
bone growth
Robert Olney
Nemours Children's Clinic, Endocrinology, Jacksonville, Florida,
United States

C-type natriuretic peptide (CNP), acting through its receptor, natriuretic peptide receptor-B (NPR-B), has been found to play a critical role in linear growth. Knockout mice for CNP and NPR-B are dwarfed, and transgenic mice overexpressing CNP are overgrown. In humans, homozygous NPR-B mutations are the cause of acromesomelic dysplasia, Maroteaux type (AMDM), a severe form of short-limbed dwarfism. Heterozygous carriers of NPR-B mutations also have reduced stature, but no other abnormalities. Hence, heterozygous NPR-B mutations are a cause of "idiopathic" short stature. Three patients have recently been described with chromosomal translocations involving breakpoints upstream of the CNP gene. Each of these patients had tall stature and evidence of CNP overexpression. Both CNP and NPR-B are expressed in the growth plate suggesting a paracrine mechanism of action. CNP acts on growth plate chondrocytes to promote terminal differentiation and hypertrophy. The CNP-NPR-B system has only recently been found to be an important regulator of human growth, and abnormalities in this system have clinical implications. Considerable work is needed to further understand this new paradigm of human growth regulation.

WG1-46 Joint Session of the ESPE Bone Club and the ESPE Growth Plate Working Group

BNP is a transcriptional target of the short stature homeobox gene SHOX

Gudrun Rappold ${ }^{1}$; Antonio Marchini'; Beate Haecker ${ }^{1}$; Tiina Marttila'; Joyce Emons' ${ }^{2}$; Birgit Weiss'; Marcel Karperien ${ }^{2}$

'Institute of Human Genetics, Human Molecular Genetics, Heidelberg, Germany; ${ }^{2}$ Leiden University Medical Center, Pediatrics and

Endocrinology and Metabolic Disease, Leiden, Netherlands

Short stature due to SHOX deficiency represents a common congenital form of growth failure and is involved in the etiology of "idiopathic" short stature and the growth deficits and skeletal anomalies in Léri Weill, Langer and Turner syndrome. While much is known on the clinical and molecular aspects of SHOX haploinsufficiency, the integration of SHOX in the signalling pathways regulating bone growth is currently not defined. Here we identify NPPB encoding the natriuretic peptide BNP, a well known approved cardiac and natriure-

47th Annual Meeting of the ESPE
WG1-47 Joint Session of the ESPE Bone Club and the ESPE Growth Plate Working Group

\section{The growth plate/bone junction}

Monika Egerbacher

Institute of Histology and Embryology, Department of Pathobiology, Vienna, Austria

Endochondral ossification involves the deposition of osteoid matrix on top of calcified cartilage and its subsequent mineralization. In the developing bone, this happens in three different sites, the primary ossification center (OC), the secondary OC and the growth plate (GP). The cellular players involved are chondrocytes and bone cells in their respective differentiation state. A relevant proportion of longitudinal and transversal growth besides cell proliferation is accomplished by hypertrophy of chondrocytes and extracellular matrix production. In the GP, chondrogenesis and osteogenesis happen at the same time and require a tightly tuned crosstalk between the chondrocytes and bone cells. The local matrix metabolism involving degradation via MMPs and resorption mediated by VEGF are essential regulators of vascular invasion and ossification during normal longitudinal growth. The close apposition of osteoblasts and chondrocytes in bone and their interaction during bone development suggest that they may each regulate the other's growth and differentiation. Previous studies have shown that both direct and indirect contact lead to reciprocal instructive interactions between co-cultered osteoblasts and chondrocytes. By cocultivation of chondrocytes and osteoblasts in RGD-containing hydrogel and subsequent implantation in immunodeficient mice, transplanted cells organized into a structure morphologically and functionally resembling growth plates. Various growth factors and hormones including FGFs, TGFs, BMPs and PTHrP are known to regulate chondrogenic differentiation but the soluble factors that mediate the specific events at the cartilage bone interface remain to be identified. The GP-bone junction comprises a functional unit where cells of the same origin work together providing mechanical stability and growth potential at the same time. Therefore, the complete system including cellmatrix and cellular interactions has to be taken into account both in clinical practice and basic research.

\section{WG1-48 Joint Session of the ESPE Bone Club and the ESPE Growth} Plate Working Group

\section{Aromatase inhibitors in bone and growth plate} Matti Hero'; Leo Dunkel

${ }^{1}$ Helsinki University Hospital, Hospital for Children and Adolescents, Helsinki, Finland; ${ }^{2}$ University of Kuopio, Department of Pediatrics, Kuopio, Finland

Without estrogen action, bone maturation is delayed, fusion of the growth plates is postponed, and longitudinal growth continues for an exceptionally long period of time. Aromatase inhibitors, blockers of estrogen biosynthesis thus offer an option for the treatment of children with short stature. However, the growth of bones (bone modeling), and the maintenance of bone (bone remodeling) are also influenced by sex steroids. Congenital lack of estrogen effects is associated with impaired accrual or maintenance of bone mass in males. The bone-preserving effects of estrogen appear to relate to its anti-resorptive influence on the bone. In a recent randomized placebo-controlled study the influence of aromatase inhibition on bone maturation, predicted adult 
height, bone turnover, cortical bone growth, and vertebral body morphology was studied in a group of peripubertal boys with idiopathic short stature (ISS) It was found that two-year treatment with the aromatase inhibitor letrozole effectively delayed bone maturation and increased predicted adult height by $5.9 \mathrm{~cm}(\mathrm{P}<0.001)$. No differences between the groups were evident in lumbar spine or femoral neck bone mineral density during the treatment, as measured by DXA. In letrozole-treated boys, the concentrations of bone resorption marker U-INTP initially increased and thereafter slowly declined, while the concentrations of bone formation markers S-PINP and S-ALP remained unchanged and slightly increased, respectively. In placebo-treated boys, all markers of bone turnover increased significantly during the treatment. Among the boys who progressed in puberty, metacarpal index (measure of cortical bone thickness) increased more in the letrozole-treated than in the placebo-treated during treatment $(25 \%$ vs. $9 \%, p=0.007)$. Mild vertebral body deformities were found in some of boys who received letrozole, but not in placebo-treated boys. Further studies are needed to evaluate the impact of aromatase inhibition on bone architecture and vertebral morphology in growing boys.

\section{WG2-49 ESPE Bone Club \\ Whole body vibration and bone mass in children with generalized cerebral palsy \\ Eckhard Schönau \\ Universitats-Kinderklinik, Köln, Germany}

Abstract text has not been submitted

\section{WG2-50 ESPE Bone Club \\ Hypophosphatemic rickets: New and important roles for bone mineralization and phosphate homeostasis of Dentin matrix protein-1 \\ Serap Turan ${ }^{1}$; Abdullah Bereket ${ }^{2}$; Tülay Güran²; Teoman Akcay²; Cumhur Aydin'; Murat Bastepe ${ }^{4}$; Harald Jüppner ${ }^{1}$ \\ ${ }^{1}$ Marmara University, Massachusetts General Hosp., Pediatric \\ Endocrinology, Endocrine Unit, Istanbul, Boston, Turkey; ${ }^{2}$ Marmara \\ University, Pediatric Endocrinology, Istanbul, Turkey; ${ }^{3}$ Gülhane Military \\ Medical Academy, Endodontics, Ankara, Turkey; ${ }^{4}$ Massachusetts \\ General Hosp. Harvard Medical School, Endocrine Unit, Boston, \\ United States}

Dentin matrix protein 1 (DMP-1), an acidic noncollagenous phosphoprotein, is a member of the SIBLING family, which includes osteopontin, bone sialoprotein, DMP-1, dentin sialophosphoprotein, enamelin, and MEPE. Each of these proteins is thought to play an important role in tissue mineralization. DMP-1 is a multifunctional protein that has been found to regulate cell attachment and cell differentiation and postulated to play a significant role in biomineralization. First data suggesting an important role of DMP-1 in bone development were obtained by studying Dmp-1 null mice. Although, Dmp-1 null embryos and newborns did not show obvious abnormality, older animals showed a profound bone phenotype characterized by shorter and wider vertebrae, and shorter long bones with delayed and malformed secondary ossification centers, an irregular and highly expanded growth plate resembling chondrodysplasia. Furthermore, Dmp-1 null animals were described as having bone features of rickets/osteomalacia and low serum phosphate $(\mathrm{P})$ levels, which provided first evidence for a role of Dmp-1 in the regulation of $\mathrm{P}$ homeostasis. Indeed, homozygous DMP-1 mutations were recently identified in the affected members of several unrelated families with an autosomal recessive form of hypophosphatemia (HP). Affected individuals show clinical, biochemical, and histomorphometric parameters that are indistinguishable from those observed in patients with X-linked HP and similar to those in autosomal dominant hypophosphatemic rickets. So far, 4 different homozygous DMP-1 mutations have been reported in 5 families; 2 families are from Lebanon and the affected individuals in both kindreds carry the same homozygous M1V mutation, which could indicate that they are related. We now describe a 6 th family, in which the affected individuals were found to have a novel homozygous frame-shift mutation (c.485Tdel; p.Glu163ArgfsX53) in exon 6 resulting in premature stop codon. Molecular findings and clinical implications in this family will be discussed in the context of bone mineralization and P homeostasis.
WG4-51 ESPE Turner Syndrome Working Group

Turner Syndrome (TS) and the Cardiovascular System

\section{Congenital heart disease and aortic dilatation evaluated by echo and MRI}

Laura Mazzanti ${ }^{1}$; Daniela Prandstraller ${ }^{2}$

'Department of Pediatrics, S.Orsola-Malpighi Hospital, University of Bologna, Bologna, Italy; ${ }^{2}$ Dept of Cardiology of Developmental Age, S.Orsola-Malpighi Hospital, Bologna, Italy

Turner syndrome (TS) is at high risk for congenital heart diseases (CHD) Recent data reports an association with a generalized vasculopathy in particular aortic dilatation (AoDil) and dissection. The complex rearrangements of Ao-root associated with AoDil are unknown.

The aim of the study was to evaluate CHD and AoDil prevalence with karyotype and dysmorphic signs. In 59 pts without CHD, followup almost 5 yrs, AoDil and the associated rearrangements were evaluated with echo and transthoracic MRI.

Methods: 682 TS enrolled by the Italian Study Group for Turner Syndrome (ISGTS) were evaluated cardiologically. MRI and echo were performed in 59 of these (12 to $28 \mathrm{yrs}$ ) without CHD and in 15 healthy volunteers. Ao-diameter [annulus, sinuses of Valsalva, sino-tubular junction (STJ) and ascending aorta (Asc-Ao)] was measured and the values were indexed for body surface area.

Results: For the pts with severe dysmorphic signs the RR of CHD was higher than for pts with minor or moderate stigmata: in particular partial anomalous pulmonary vein drainage (PPAVD), coarctation of the aorta (COA) and also bicuspid aortic valve (BAV). The $45, \mathrm{X}$ and $\mathrm{Y}$-mosaicism pts had a significantly higher prevalence of severe CHD than the other groups. 59 pts without CHD evaluated with echo and MRI had a larger annulus $(\mathrm{p}=0.004)$, STJ $(\mathrm{p}=0.0001)$, and Asc-Ao diameters $(\mathrm{p}=0.01)$ than controls. STJ dilation was observed in $11 / 59 \mathrm{pts}(18.6 \%)$ at echo and in $8 / 59 \mathrm{pts}(13.6 \%)$ at MRI. Asc-Ao dilation was observed in $9 / 59$ pts $(15.2 \%)$ at echo and in $7 / 59$ pts $(11.9 \%)$ at MRI. TS patients with a dilated Asc-Ao displayed a complex rearrangement of Ao with prominent dilation of the STJ.

Conclusions: Our data confirm the high risk for CHD and AoDil in TS; a severe phenotype is a risk factor. Dilation of Asc-Ao and STJ is relatively common in TS with a reshaping of the Ao-root. An accurate echo follow-up is highly recommendable even in absence of CHD. Also with a good agreement in Ao-image between echo and MRI, we underline the importance of MRI to detect better AoDil in all TS over 15 yrs of age.

\section{WG4-52 ESPE Turner Syndrome Working Group}

Turner Syndrome (TS) and the Cardiovascular System

\section{Cardiovascular events in Turner syndrome \\ Britta Hjerrild}

Aarhus University Hospital, Department of Endocrinology and

Diabetes, Aarhus, Denmark

Overall mortality is increased in women with Turner syndrome (TS) with a standardized mortality rate (SMR) of 2.9. This is partly explained by a significantly increased risk of premature cardiovascular death on the basis of a high prevalence of congenital cardiovascular malformations $(\mathrm{SMR}=24)$ and an increased incidence of aortic dilatation and dissection of 40/100.000 in TS compared to $6 / 100.000$ in the background population. In addition aortic dilatation and dissection occur much earlier, at a median age of 35 years in TS compared to 71 years in the background population. Furthermore hypertension is described in both girls and women with TS, with an abnormal diurnal blood pressure profile (nocturnal "non dippers") and a prevalence of hypertension of around $50 \%$ in adult TS populations, all contributory factors to the increased risk of secondary ischemic heart disease and stroke has been found. Discrete left ventricular systolic and diastolic dysfunction in young normotensive women with TS and no cardiac symptoms has been found by tissue doppler echocardiography. In addition, the TS patient group has an elevated body mass index, increased body fat mass, decreased muscle mass as well as excess visceral fat compared to controls. Epidemiological data find that diabetes as well as hypothyroidism is more frequent in TS, and find an increased mortality due to endocrine diseases $(\mathrm{SMR}=5.7)$. All factors that can potentiate the spectrum of risk factors for developing cardiovascular disease in TS So the importance of monitoring the heart, aorta as well as metabolic parameters in this patient group has become increasingly clear and calls for a life-long, multi-disciplinary care for women with TS. 
WG4-53 ESPE Turner Syndrome Working Group

Turner Syndrome (TS) and the Cardiovascular System

The role of paediatric endocrinologist in ameliorating adult cardiovascular pathology

\section{in TS}

Kerstin Albertsson-Wikland

Gotheburg, Sweden

Abtstract text has not been submitted

WG4-54 ESPE Turner Syndrome Working Group

Turner Syndrome (TS) and the Cardiovascular System

Transition of TS patients from paediatric to adult care

\section{Sabine de Muinck Keizer-Schrama}

ErasmusMC/Sophia Children's Hospital, pediatric-endocrinology,

Rotterdam 3015 GJ, Netherlands

Turner's syndrome (TS) is the most common chromosomal abnormality in females, affecting 1: 2500 live female births. It is the result of complete or partial absence of one $\mathrm{X}$ chromosome. The most consistent clinical features are short stature and ovarian failure. Adults with TS have a reduced life expectancy, mainly due to excess cardiovascular risk. They may also have multiple co morbidities including hypothyroidism, skeletal problems, renal and gastrointestinal disease, hearing loss, and psychosocial problems. Thereby they are more prone to develop type 2 diabetes and dyslipidemia. While most females with TS present during childhood and adolescence, one quarter are diagnosed in adulthood with primary or secondary amenorrhoea. Girls with TS usually receive intensive medical care during childhood. Early diagnosis is mandatory not only for achievement of optimal adult height and age-appropriate puberty induction but also for prevention and treatment of co morbidities. This requires a multidisciplinary approach and a protocol from the Dutch Advisory Group on Growth Hormone is presented. These aspects also provide the rationale for a structured transition of these patients. Transition is an appropriate time to assess individual risks for potential adult morbidities and promote healthy life-styles. The emphasis throughout transition to adult care is on educating the adolescent with TS, her family, and the receiving adult care providers. Several follow-up studies indicated that the necessary continuum of care is not optimal. Recommendations for the transition and adult care of women with TS will be given with emphasis on a multidisciplinary approach. syndrome. Given the significant increase in waist circumference among children and adolescents over the last two decades, a marker of abdominal obesity should be considered as an important component of the pediatric metabolic syndrome definition. Moreover, if one is to develop a metabolic syndrome criteria that can be used worldwide, a marker of abdominal obesity could be more useful than BMI since certain populations have higher abdominal adiposity than others for a given BMI. Much research is needed to establish the clinical translation of childhood metabolic syndrome for the future risk of T2DM and CVD.

\section{WG5-56 ESPE Obesity Club The Metabolic Syndrome in the Paediatric Age Range - Towards International Consensus \\ Systems biology and childhood obesity Ze'ev Hochberg'; Annette Gruters-Kieslich ${ }^{2}$ \\ ${ }^{1}$ Meyer Children's Hospital, Endocrinology, Haifa, Israel; ${ }^{2}$ Charite, Pediatrics, Berlin, Germany}

Obesity is a complex condition involving many different pathways on the genetic, cell, tissue and organ level, and only the application of modern bioinformatics and the identification of important submodules, nodes and hubs of this network will enable us to understand the mechanisms involved in the manifestation of childhood obesity and associated comorbidities To apply these data sets to reveal the operation of significant biological networks in childhood obesity several circuits are considered. The social and environmental circuit plays a direct role in children's eating patterns through their behaviors, attitudes, and feeding styles. Childhood obesity is highly heritable; The candidate gene approach has identified very few monogenic diseases, all linked to the central leptin - melanocortin pathway. Several whole genome association studies identify further genes associated with small but significant differences in the BMI of obese compared to non-obese cohorts. Energy conserving networks involve interaction of adipose tissue, hormonal and neural pathways that regulate food intake and body fat mass. Once obesity develops, changes in cell-intrinsic circuits have negative impact on insulin signaling, leading to ectopic fat storage and an increase in fatty acids, reactive oxygen species generation by mitochondrial oxidation, and mitochondrial dysfunction. Quantitative screening of probe-proteome reactions exposed several enzyme activities differentially expressed in lean and obese mice, discovering the hydroxypyruvate reductase pathway. At the metabolomics levels, a profound decrease in the ATP/ADP ratio in the liver tissue was found in obese Zucker rats, and a marked increase in methionine and decrease in betaine was detected. Future studies of gene expression, protein signatures, protein-protein interactions and metabolic signatures in cells and tissues will be important to understand how different types of obesity as well as comorbidities can be explained.

\section{WG5-55 ESPE Obesity Club The Metabolic Syndrome in the Paediatric Age Range - Towards International Consensus The metabolic syndrome in the pediatric age
range Silva Arslanian \\ Children's Hospital of Pittsburgh of UPMC, Weight Management \& Wellness Center, Pittsburgh, United States}

The metabolic syndrome is a cluster of metabolic abnormalities characterized by insulin resistance/hyperinsulinemia, glucose intolerance, Low HDL, high triglycerides and hypertension, and is believed to play an important role in the development of type 2 diabetes (T2DM) and atherosclerotic cardiovascular disease (CVD). In adults, various definitions of the metabolic syndrome have been proposed . In pediatrics there are no uniformly agreed upon criteria for the metabolic syndrome. Recently the International Diabetes Foundation (IDF) published criteria for the metabolic syndrome in children . During this lecture pediatric data will be presented that: 1) obesity is the driving force for the pediatric metabolic syndrome since the prevalence is substantially lower in normal weight ( $1 \sim 3 \%)$ compared with overweight ( $24 \sim 51 \%)$ youth; 2$)$ the prevalence of the individual components of the metabolic syndrome increases with decreasing in vivo insulin sensitivity, 3 ) regardless of the metabolic syndrome criteria used, youth with the metabolic syndrome are characterized by increased waist circumference and visceral adiposity, insulin resistance, hyperinsulinemia, and hypoadiponectinemia; 4) in children with the metabolic syndrome, IL-6 and endothelial biomarkers (ICAM and E-selectin) are elevated; 5) waist circumference predicts individual components of the metabolic

\author{
WG5-57 ESPE Obesity Club The Metabolic Syndrome in the \\ Paediatric Age Range - Towards International Consensus
Gene - environment interaction in relation to obesity and related traits \\ Ruth J.F. Loos \\ MRC Epidemiology Unit, Institute of Metabolic Science, Cambridge, \\ United Kingdom
}

A changing environment that promotes excessive calorie intake and that discourages physical activity appears to be the main culprit of the dramatic increase in obesity prevalence worldwide over the past three decades. However, this obesogenic environment does not affect every person to the same extend, highlighting the variation in susceptibility to weight gain that is likely to be genetically determined. Indeed, obesity is a common, multifactorial disease that arises through the joint actions of multiple genetic and environmental factors. Although it seems obvious to propose a gene-lifestyle interaction model for the development of obesity, it is altogether not straightforward to identify the molecular mechanisms that underlie such a model. Neither is it easy to identify such a model in epidemiological studies. In this presentation, I will review the basic methodological principles and epidemiological study designs that characterize good gene-lifestyle interaction studies. While direct gene effect studies require substantial sample sizes to identify the (small) influences on obesity risk and related traits, even larger sample sizes are required when testing for gene-lifestyle interaction. Critical for the success of gene-lifestyle studies is accuracy of the environmental measures, such as food intake and 
physical activity. I will review how study design and accuracy of measurement affect power to identify such interactions, illustrated by classical and also more recent examples from obesity research. The ultimate endeavour for gene-lifestyle interaction studies is to translate these new 'interaction' insights into clinical practice. As with pharmacogenetics, it is expected that results from gene-lifestyle interaction studies will lead to personalized treatment and lifestyle prescription. I will briefly discuss how close (or far) we are to this ambitious goal and make comparison to successes in pharmacogenetics.

WG5-58 ESPE Obesity Club The Metabolic Syndrome in the Paediatric Age Range - Towards International Consensus

The Metabolic syndrome in the paediatric age range - a future perspective

Claire Levy-Marchal

Hopital Robert Debre, Paris, France

Abstract text has not been submitted

\section{WG6-59 ESPE Disorder of Sex Development (DSD) Working Group The development of ESPE DSD registry Faisal Ahmed \\ Glasgow, United Kingdom}

Abstract text has not been submitted

\section{WG6-60 ESPE Disorder of Sex Development (DSD) Working Group Development of an e.learning web portal on DSD}

Stenvert L.S. Drop; K Grijpink; Ivan Oosterhout

Sophia Children's Hospital, ErasmusMC, Div ped Endocrinology,

Rotterdam, Netherlands

Based on experience gained with an interactive program: "Pediatric Endocrinology IN ter ACTION, Growth and Puberty" (ErasmusMC;2004), presently available on the website of ESPE, LWPES and SLAP and containing core modules aimed at medical students and advanced modules aimed at post-doc students, fellows etc, an editorial and technical staff has been formed to develop an e.learning web portal to give entrance to an interactive learning environment for an up to date program on DSD including normal development, patho-physiological mechanisms and current views on diagnostic and therapeutic interventions, psychological counseling, outcome. Furthermore, study results will be implemented and evidence based guidelines provided. It will be a meeting place of experts, teachers and students at various levels, to gain, share, contribute and develop knowledge in an accessible and flexible way. It will allow for assessment of several competencies. The "audience" will consist not only of ESPE members but the program will also reach out to health care workers globally.The e-learning portal will contain several functionalities: a) Personal login, account and access rights; b) Theoretical information offered in a number of chapters covering relevant theory and cases and will include images, animations, video and questions aimed at developing several competencies; c) Mediclopedia', offering the user the option to lookup specific subjects in an alphabetically ordered list; d) Forum, enabling the users to post comments and remarks, to discuss and to share knowledge; e) Assessment, allowing for assessment of various competencies; f) Administrative general information, content management, search. A board of medical editorial, educational and technical contributors has been formed.

\section{WG6-61 ESPE Disorder of Sex Development (DSD) Working Group From national networks to a European collaborative study on DSD}

\section{Olaf Hiort}

University of Lübeck, Department of Pediatrics, Lübeck, Germany

Disorders of Sex Development (DSD) constitute an array of rare to very rare disorders affecting the genito-urinary tract and in most instances also the endocrine-reproductive system. To date, the aetiology of DSD in many of the patients remains unresolved. Therefore, novel approaches to the diagnosis are crucially needed and are very likely to provide important novel insights into the aetiology of DSD, which will translate into clinical benefits by improving the diagnostic assessment of patients, the basis for prognostic predictions and eventually by groundbreaking for novel treatments and individually tailored therapeutic approaches. The German government established a programme for networks on rare diseases, funding 10 nationally operating networks from 2003 until 2008. The German Network on DSD (www.netzwerk-is.de) addresses the development of structures for research on rare disorders of sex development with regard to basic research related to defining genetic disorders, assessment of intracellular steroid metabolism in well characterized disorders, and a large clinical evaluation study. However, several issues of genetic, biochemical and functional studies cannot be addressed due to the restriction of the network to the necessarily smaller, national patient cohorts. In addition, the availability of specialised expertise in DSD is limited in comparison to the options that cross-European collaboration offers in the DSD field. This has led to a collaborative approach of six European countries for a structured reseach project called EuroDSD to gain funding within the 7th European Framework Programme. EuroDSD starts in May 2008 for a period of 3 years by linking a European patient-based data collection and analysis tools with research on development of novel diagnostic strategies to identify new causes of DSD in conjunction with a strong programme on functional molecular biology of the androgen receptor, thereby allowing for an in-depth analysis of a key factor in the pathogenesis of DSD.

\section{WG6-62 ESPE Disorder of Sex Development (DSD) Working Group Consequences of the Chicago consensus on DSD: current practice in Europe}

leuan A. Hughes

Cambridge, United Kingdom

Abstract text has not been submitted

\section{WG6-63 ESPE Disorder of Sex Development (DSD) Working Group The molecular biology of congenital adrenal hyperplasia in the Mediterranean Area \\ Antonio Balsamo; Lilia Baldazzi; Soara Menabò; Alessandro Cicognani Univ. of Bologna and S.Orsola-Malpighi Hospital, Dpt. for Woman, \\ Child and Adolescent Health, Bologna, Italy}

Congenital adrenal hyperplasia $(\mathrm{CAH})$ due to 21-hydroxylase deficiency (21OHD) is one of the most common autosomal recessive metabolic diseases and occurs worldwide. Ethnic-specific differences in the distribution of CYP21A2 gene mutations have been described so far. In this report we focused our attention on the Mediterranean area and compared the types and the relative frequencies of molecular defects in 233 unrelated patients of Italian origin (as defined by review of pedigrees extending to two or three generations) with the reported frequencies in other "Mediterranean" Countries. In particular, the distribution of mutations were available for samples of Croatian, French, Hellenic, Lebanese, Slovenian, Spanish, Tunisian, and Turkish populations. The predominance of the allelic mutations and genotypes in some of these populations was significant and ethnic- or intra-ethnic specific mutations were found. A large deletion is prevalent in the Slovenjans; a Q318X mutation is prevalent in the Tunisians; a R356W mutation is prevalent in the Croatians; the D8bp mutation was quite frequent in the Lebanese population exclusively in the Christian Maronite group. Intra-National differences were also found when the comparison was extended to groups coming from different areas of the same Nation such as North-West vs. Central Spain or North-Central vs. South Italy. The more frequent genotype/phenotype discrepancies will be discussed. 
WG6-64 ESPE Disorder of Sex Development (DSD) Working Group Testis development: update

Olle Söder

Stockholm, Sweden

Abstract text has not been submitted

WG6-65 ESPE Disorder of Sex Development (DSD) Working Group 46,XY DSD with a normal testicular function Yves Morel

Lyon, France

Abstract text has not been submitted

\section{WG6-66 ESPE Disorder of Sex Development (DSD) Working Group Molecular diagnoses in a series of 122 index patients with $46, X Y$ disorder of sex development}

Laura Audí'; Mónica Fernández-Cancio'; Carmen Piró2; Nùria Torán ${ }^{3}$; Pilar Andaluz'; Pilar Andaluz'; Miquel Gussinyé ${ }^{1}$; Antonio Carrascosa ${ }^{1} H o s p i t a l$ Vall d'Hebron, CIBERER, UAB, Paediatric Endocrinology Research Unit, Barcelona, Spain; ${ }^{2}$ Hospital Vall d'Hebron, CIBERER, UAB, Paediatric Urology Surgery, Barcelona, Spain; ${ }^{3}$ Hospital Vall d'Hebron, CIBERER, UAB, Pathology Department, Barcelona, Spain

A series of 122 index patients, referred from our centre and 30 other Spanish hospitals, presenting a 46,XY disorder of sex development (46,XY DSD) were analysed for a molecular diagnosis in the last six years. Genes analysed were: AR (androgen receptor), SRD5A2 (5 $\alpha$-reductase type 2), HSD17B3 (17ß-hydroxy-steroid dehydrogenase type 3), CYP17A1 (17 $\alpha$-hydroxylase and 17,20-lyase) and LHCGR (LH/CG receptor). A molecular diagnosis was reached in $61.5 \%$ of patients, with anomalies corresponding to the following genes or chromosomes: AR (45.9\%), SRD5A2 (6.5\%), HSD17B3 (3.3\%), CYP17A1 (0.9\%), LHCGR (0.9\%), Denys-Drash syndrome, other testicular dysgenesis syndromes (9-del-p23-pter and $18 \mathrm{p}$-del) $(3.2 \%)$ and septo-optic dysplasia $(0.8 \%)$, while in $38.5 \%$ of patients no molecular diagnosis was obtained. Among the phenotypes, $38.5 \%$ were completely female and $61.5 \%$ ambiguous. Among patients with female genitalia, a molecular diagnosis was reached in $91.5 \%$ of cases, with $89.4 \%$ carrying one or two (in 2 cases) AR gene mutations, $2.1 \%$ CYP17A1 mutations, and no affected gene was detected in $8.5 \%$. Among the ambiguous genitalia, a molecular diagnosis was obtained in only $42.6 \%$ of cases, with $18.6 \%$ carrying an AR mutation, $10.6 \%$ SRD5A2 mutations, 5.4\% HSD17B3 mutations, $1.4 \%$ LHCGR mutations, $5.3 \%$ testicular dysgenesis of descriptible origin and $1.3 \%$ septo-optic dysplasia. In conclusion, detection of the molecular cause of $46, \mathrm{XY}$ DSD was successful in $61.5 \%$ of patients, with AR gene being the most frequently affected; however, the percentage was higher in the completely female phenotype, as was that of an affected AR gene. Thirty-eight percent of patients remained without a molecular diagnosis owing to lack of adequate biochemical and/or candidate gene orientation or to the lack of analysis of new candidate genes still to be described.

\section{WG6-67 ESPE Disorder of Sex Development (DSD) Working Group RNA-array technology and its use in defining sex-related gene expression \\ Paul-Martin Holterhus \\ Chrsitian Albrechts University of Kiel, Department of Pediatrics, 24105 Kiel, Germany}

Normal male external genital differentiation is dependent on normal androgen action via the androgen receptor (AR). Mutations in the AR-gene inhibit ARfunction and in turn lead to androgen insensitivity syndrome (AIS) in 46,XY individuals, one of the most common disorders of sex development (DSD). Clinical management would benefit from markers of individual AR-function. Moreover, many patients with presumed AIS on clinical grounds don't seem to have mutations in the AR-gene. This talk will review our recent genomewide studies on AR-function and sex-specific gene expression in genital skin fibroblast cultures using cDNA-microarrays. Within the external genitalia, fibroblasts contain a stable gene transcription signature which represents their origin of biopsy. Therefore, only homologous genital fibroblasts may be compared with respect to AR-function and sex-specific gene expression. If performed this way, fibroblasts derived from labia majora in 46,XY CAIS patients having documented AR-mutations and from normal scrotum (both originated from the embryonic urogenital swellings) differ significantly in mRNA transcription of 612 transcripts. Inclusion of independent samples derived from complete and partial AIS (CAIS and PAIS, respectively) patients confirms that this gene transcription signature represents a "transcriptional memory" of prenatal genital androgenization. The identified genes will be helpful to decipher mechanisms of external genital development. In a very recent unpublished microarray - and RT-PCR study, DHT-treatment of normal scrotum fibroblasts revealed significant up-regulation of Apolipoprotein D, a regulator of senescence and an important pheromone transporter in humans. CAIS patients did not respond, PAIS did respond marginally while normal scrotum-like up-regulation was present in labia majora of 17ßHSD type III deficiency indicating AR-specificity. Therefore, in addition to analysis of individual androgen memory, functional analysis of APOD in genital fibroblasts could provide promising insights into AR-function of an individual with DSD. 